\title{
THE FAINT END OF THE LUMINOSITY FUNCTION OF GALAXIES IN HICKSON GROUPS
}

\author{
STEPHEN E. ZePF ${ }^{1}$ \\ Department of Astronomy, University of California, Berkeley, Berkeley, CA 94720, and Department of Astronomy, \\ Yale University, New Haven, CT 06520; zepf@astro.yale.edu \\ Reinaldo R. De Carvalho \\ Observatório Nacional, Conselho Nacional de Desenvolvimento Científico e Tecnologico, Departamento de Astrofísica, Brazil \\ AND \\ ANDRÉ L. B. RiBEIRO \\ Divisão de Astrofísica, Instituto Nacional de Pesquisas Espaciais, Caixa Postal 515-12201-970, São José dos Campos, Brazil \\ Received 1997 June 2; accepted 1997 July 30; published 1997 September 5
}

\begin{abstract}
We study the luminosity function of galaxies in Hickson groups using our recent redshift survey of galaxies in and around 17 of these groups. We find that the galaxies in these regions have a luminosity function with $M_{*}=-19.5+5 \log h$ and $\alpha=-1.0$, where $M_{*}$ and $\alpha$ are the usual parameters in the standard Schechter form of the luminosity function and the magnitudes are measured in the $B$ band. The formal $95 \%$ confidence intervals for $M_{*}$ and $\alpha$ range from $(-19.3,-0.8)$ to $(-19.7,-1.2)$ and are highly correlated, as is usual for these fits. This luminosity function for galaxies in our Hickson group sample is very similar to that found in large surveys covering a range of environments. These values are also consistent with our earlier estimates based on a photometric analysis with statistical background correction and do not support previous suggestions of an underabundance of intrinsically faint galaxies in compact groups. We confirm our earlier finding that the fainter galaxies are more diffusely distributed within individual groups than the brighter ones. This can be interpreted either as evidence for mass segregation within the groups or as the result of the selection procedure for Hickson groups.
\end{abstract}

Subject headings: galaxies: clusters: general — galaxies: interactions galaxies: luminosity function, mass function - galaxies: statistics

\section{INTRODUCTION}

The luminosity function of galaxies is generally described by a function of the form

$$
n(L) d L \propto e^{-L / L_{*}}\left(L / L_{*}\right)^{\alpha} d L
$$

(Schechter 1976). However, there is little consensus on the value of $\alpha$, the slope of the faint end of the luminosity function. Published values range from roughly $\alpha \simeq-0.8$ to $\alpha \simeq-2.0$. Moreover, it is not yet clear whether the slope of the faint end of the luminosity function is dependent on the environment of the galaxies sampled.

Possible environmental variations of the faint-end slope of the galaxy luminosity function are interesting, as they may reflect the astrophysical process during galaxy formation or the subsequent dynamical evolution. Observations suggest that higher luminosity galaxies are more strongly clustered than low-luminosity galaxies (e.g., Lin et al. 1996a; Loveday et al. 1995). This is at least qualitatively consistent with the predictions of biased galaxy formation models in which low-density regions preferentially harbor low-luminosity galaxies (e.g., White et al. 1987). Dynamical effects may also lead to environmental differences in the luminosity function. For example, mass segregation will tend to lead toward more massive objects in denser regions. However, these massive galaxies may also preferentially disappear through merging as the result of dynamical friction.

In practice, the slope of the faint end of the luminosity function is determined in one of two ways. The most straightforward approach is through large redshift surveys. The largest and most recent of these is the Las Campanas Redshift Survey (LCRS),

${ }^{1}$ Hubble Fellow. from which Lin et al. (1996b) derive $\alpha=-0.70 \pm 0.05$ for galaxies in the magnitude range $-22 \gtrsim M_{B}+5 \log \mathrm{h} \gtrsim$ -16.5 , with evidence for a somewhat steeper slope $(\alpha \simeq-1)$ when the fit is extended to fainter galaxies. The LCRS luminosity function is in good agreement with that derived from the earlier Stromlo-Automatic Plate Measuring Facility survey, for which Loveday et al. (1992) found $\alpha \simeq-1$. A similarly shallow slope was found in the CfA survey for brighter magnitudes (Marzke et al. 1994a). However, Marzke et al. (1994a) also found a significant steepening of the slope at the faint end that is not seen in either of the other surveys.

An alternative technique is to compare the galaxy counts in a cluster to those outside the cluster and thereby derive statistically a luminosity function for the cluster. This approach has now been applied to a number of clusters, with varied results. For example, Gaidos (1997) surveyed 20 Abell clusters and found $\alpha \simeq-1.1$, consistent with earlier results of Schechter (1976) and Dressler (1978), who found fairly flat slopes for the luminosity function of cluster galaxies. Detailed studies of the Coma Cluster (Bernstein et al. 1995) and the Virgo Cluster (Sandage, Binggeli, \& Tammann 1985) reveal somewhat steeper slopes, with $\alpha \simeq-1.4$. Much steeper slopes $(\alpha \simeq-2)$ were found by De Propis et al. (1995) for four low-redshift clusters and by Driver et al. (1994) for several clusters at moderate redshift. Lopez-Cruz et al. (1997) claim a systematic variation from shallow to steep slopes with decreasing richness of clusters, consistent with the early work of Oemler (1974), although other studies have indicated a universal luminosity function for cluster galaxies (e.g., Lugger 1986; Colless 1988).

One effective way to test for environmental effects on galaxies is to study galaxies in Hickson groups. These groups were selected on the basis on their very high surface densities (Hickson 1982). Subsequent spectroscopic observations have 


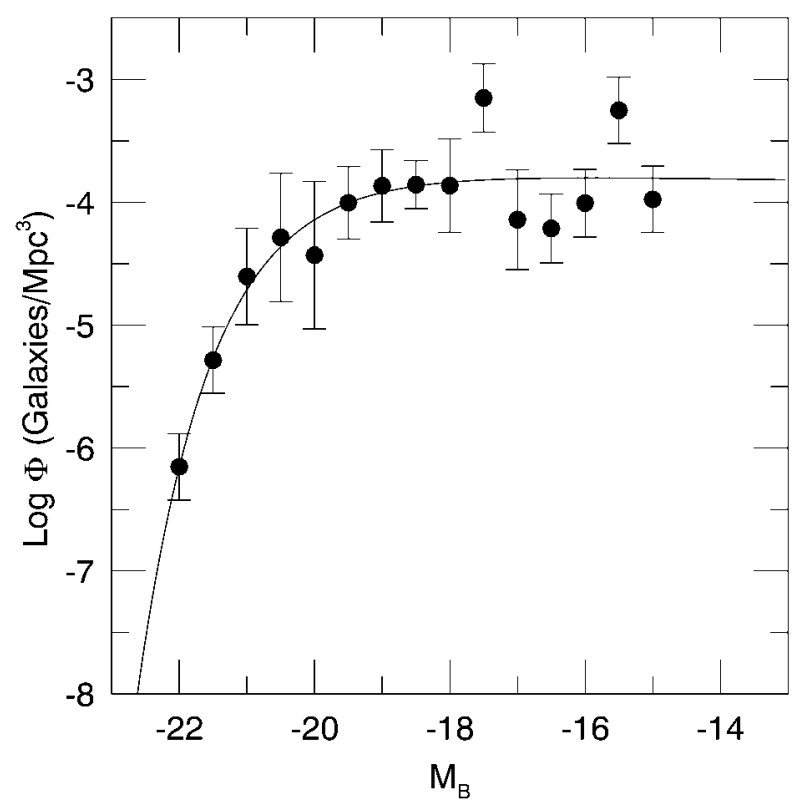

FIG. 1.-Plot of the luminosity function of galaxies in our sample of 17 nearby Hickson groups. The best-fitting Schechter luminosity function is shown as the solid line. The data clearly indicate a flat slope for the faint end of the luminosity function $(\alpha \simeq-1)$. We adopt $H_{0}=75 \mathrm{~km} \mathrm{~s}^{-1} \mathrm{Mpc}^{-1}$ to determine absolute magnitudes for this and subsequent plots.

established that most of the galaxies in individual groups are at similar redshifts and that the groups typically have velocity dispersions of 100-350 $\mathrm{km} \mathrm{s}^{-1}$ (Hickson et al. 1992; see also Ribeiro et al. 1997). The combination of high spatial densities inferred from the projected galaxy distribution and velocity dispersions similar to the internal velocities of galaxies gives short timescales for dynamical evolution through galaxy merging in compact groups, as dramatically demonstrated by Barnes (1989).

Given the short timescales for dynamical evolution expected in Hickson groups, it is interesting to compare the luminosity function of galaxies in Hickson groups with that of the general field population. We previously addressed this problem by counting galaxies in and around a sample of Hickson groups and then statistically correcting for background galaxies ( $\mathrm{Ri}$ beiro, de Carvalho, \& Zepf 1994). This leads to an estimate of the faint end of the galaxy luminosity function in much the same way as the cluster studies described above. With this approach, we were able to reach much fainter magnitudes than those considered by Hickson in his group selection, thereby avoiding the difficult problem of accurately modeling the selection effects in the Hickson sample that led to disagreements among earlier studies (Mendes de Oliveira \& Hickson 1991; Sulentic \& Rabaca 1994). Reaching fainter magnitudes is also obviously valuable for improving the leverage on the determination of the slope of the faint end of the luminosity function.

The photometric analysis indicated that the faint end of the luminosity function was well fitted by a Schechter function with $\alpha=-0.82 \pm 0.09$. The uncertainty reflects the statistical uncertainty in the number of galaxies detected above the estimated background. There are potential systematic concerns associated with the background corrections. Therefore, one of the motivations for our spectroscopic survey of faint galaxies in and around compact groups (de Carvalho et al. 1997) was to eliminate the need for statistical background correction by obtaining redshifts for these galaxies. This Letter reports the results of the analysis in $\S 2$ and discusses the implications of these results in $\S 3$.

\section{ANALYSIS}

In order to determine the luminosity function of compact groups, we utilize redshifts determined in our spectroscopic survey of galaxies in and around 17 Hickson groups (de Carvalho et al. 1997). $B$ magnitudes are obtained from our earlier photometric analysis of galaxies in these regions (de Carvalho, Ribeiro, \& Zepf 1994). We then combine the redshifts and the photometry to determine the distribution of galaxy luminosities in each group. The faint limit of this procedure is taken to be the $B$ magnitude at which our redshift survey is $10 \%$ incomplete for that group. In order to determine the luminosity function, we weight the galaxy luminosity distribution in each group by the effective volume $\left(v / v_{\max }\right)$ of that group. For the selection function of the groups, we adopt the form $P(m)=[1+$ $\left.10^{1.2\left(m-m_{0}\right)}\right]^{-1}$ given by Hickson, Kindl, \& Aumann (1989) for the Hickson group sample. As described in Ribeiro et al. (1994), $m_{0}=13.0$ gives the best fit to the cumulative distribution of total magnitude of our sample of groups, which is a subsample of the total Hickson catalog (de Carvalho et al. 1994). The galaxy luminosity function for the sample as a whole is then determined by a straightforward summation over the 17 groups by using the $v / v_{\max }$ weighting for each group. Because each group encompasses a wide range of galaxy luminosities, the shape of the resulting galaxy luminosity function is not sensitive to the details of the weighting procedure. Uncertainties in the effective volume of different groups tend to shift the normalization of the luminosity function $\left(\phi^{*}\right)$ but not its shape $\left(M_{*}\right.$ and $\left.\alpha\right)$.

The resulting luminosity function for galaxies in our sample of 17 Hickson groups is given in Figure 1. Also plotted in this figure is the best-fitting Schechter function, which has $M_{*}=$ $-19.5+5 \log h$ and $\alpha=-1.0$, as well as $\phi^{*}=2 \times 10^{-4}$. The parameters, $\phi^{*}, M_{*}$, and $\alpha$, were determined by a nonlinear least-squares fit (Jeffreys, Fitzpatrick, \& McArthur 1988). The error bars for the individual points were determined by the standard deviation $(1 \sigma)$ of the galaxy counts in each luminosity bin.

As is usual for these fits, the values of $M_{*}$ and $\alpha$ are highly correlated, and the formal $95 \%$ confidence limits on $M_{*}, \alpha$ combinations are $(-19.7,-1.2)$ and $(-19.3,-0.8)$.

The primary result of this Letter is that the luminosity function of galaxies in our sample of Hickson groups is very similar to that found in similar surveys of large samples of galaxies covering a wide range of environments. This agreement is shown in Figure 2, where we plot both our luminosity function for galaxies in Hickson groups and the LCRS luminosity function of Lin et al. (1996b). The $r$ magnitudes of the LCRS have been converted to $B$ magnitudes using $B-r=1.1$ (Lin et al. 1996b). The luminosity functions have been offset arbitrarily in the $y$-axis for ease of comparison.

We also note that the luminosity function derived here from our spectroscopic survey is consistent with the one we derived earlier by comparison galaxy counts inside and outside the groups (Ribeiro et al. 1994). This agreement suggests that the statistical background subtraction adopted in our earlier paper is reliable. It also suggests that statistical techniques on photometry around compact groups could be applied to many more groups to improve statistics and to look for systematic trends with group properties. Furthermore, the spectroscopic data confirm the conclusion of our photometric analysis that the faint 


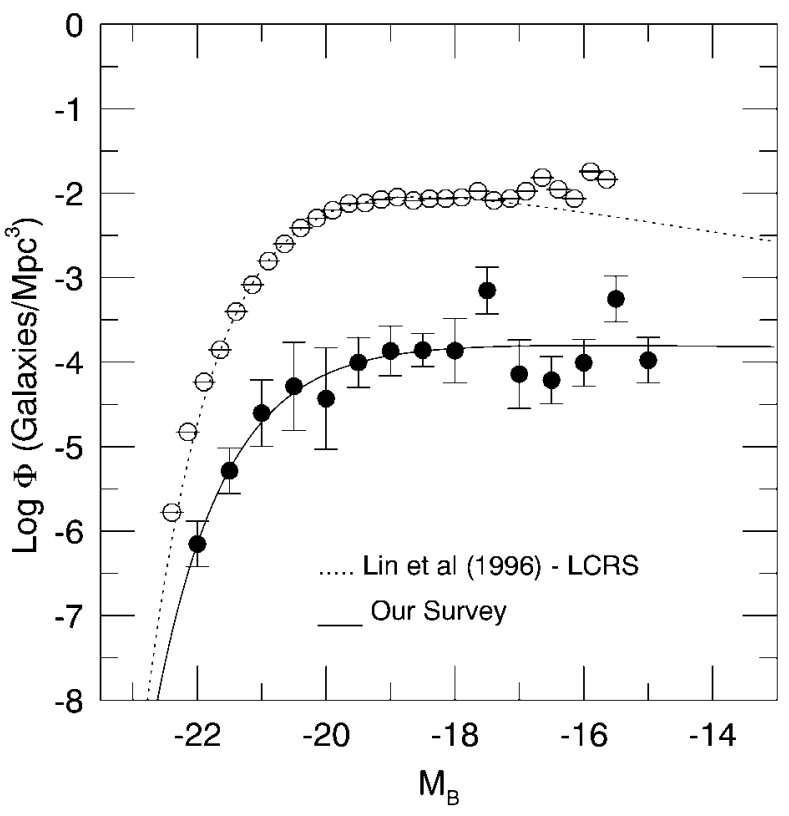

FIG. 2.-Comparison of the luminosity function derived for our sample of galaxies in Hickson groups with that found by Lin et al. (1996b) for the large LCRS. They appear to have a very similar shape. The vertical offset is arbitrary.

galaxies in our Hickson group sample are more diffusely distributed than the brighter galaxies (Ribeiro et al. 1997). The implications of this result are discussed in the following section.

Possible differences between the luminosity functions of various types of galaxies are also of interest. In spectroscopic surveys like ours, a natural division is between galaxies with and without emission lines. In Figure 3, we plot the luminosity function for galaxies in our Hickson group sample, with galaxies in which we detect emission lines now plotted with different symbols than those in which we do not detect emission lines. This figure shows that the luminosity function for emission-line galaxies appears to be shallower than that for galaxies without emission lines.

If confirmed, this result would indicate a difference between galaxies in Hickson groups and those in the general field, as Lin et al. (1996b) found that emission-line galaxies have a steeper faint-end slope than galaxies without emission lines. Similarly, Loveday et al. (1992) found that galaxies classified as early type (less likely to have emission lines) have shallower faint-end slopes than those classified as later type. Although this latter result may have been affected by the difficulty of classifying galaxies on the available plate material (Marzke et al. 1994b), no previous redshift survey has found that galaxies with emission lines or of later morphological type have a shallower faint-end slope than galaxies without emission lines or of earlier type. A result that might be similar to ours is that Sandage et al. (1985) find that the very faint end of the luminosity function in Virgo is dominated by dwarf ellipticals, which are not known to have emission lines.

A concern in the comparison of our Hickson group galaxy luminosity function for emission- and nonemission-line galaxies and other surveys is whether the classification of galaxy spectral type is similar. In our survey, galaxies are classified as emission-line objects if the equivalent width of $\mathrm{H} \alpha$ is greater than $6 \AA$. Approximately $60 \%$ of the galaxies in our Hickson groups sample are classified as having emission lines on this basis. As a comparison, in the red-selected LCRS, roughly $50 \%$

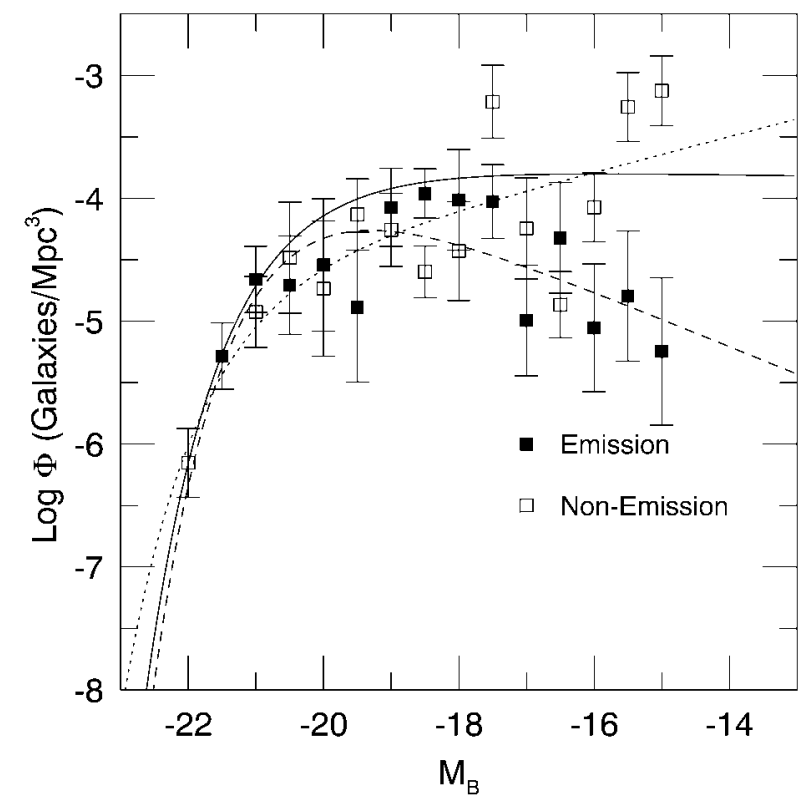

FIG. 3.-Plot of the luminosity function of our sample of galaxies in Hickson groups, divided by the presence or absence of emission lines in the spectrum of the galaxy, where a galaxy is said to have emission lines if $\mathrm{EW}(\mathrm{H} \alpha)>$ $6 \AA$. This figure shows a decline in the number of emission-line galaxies at faint magnitudes. Similar surveys of galaxies in other environments typically find the opposite trend. If confirmed, this result represents a significant difference between galaxies in compact groups and those in other environments.

of the full sample of galaxies are classified as emission-line objects, on the basis of having [O II] equivalent widths of more than $5 \AA$. In the blue-selected samples of Marzke et al. (1994b) and Loveday et al. (1992), about $70 \%$ of the galaxies are classified as late type on the basis of morphology. Hickson groups are known to be somewhat more elliptical rich than those in the general field (see, e.g., Hickson, Kindl, \& Huchra 1988). Therefore, the identification of emission-line objects in our blue-selected Hickson sample is at least roughly consistent with that in other samples. This suggests that the spectral classification itself is not responsible for the observed differences in luminosity function as a function of spectral type between our Hickson group sample and field galaxy samples.

\section{DISCUSSION}

The primary conclusion of this Letter is that the faint end of the luminosity function of galaxies in Hickson groups is similar to that found in general field surveys. This result fits well into the picture that most galaxies in Hickson groups are not significantly different from those in other environments. There is good evidence for enhanced merging activity in compact groups (Zepf 1993, and references therein) and peculiarities in the isophotal properties of Hickson group ellipticals that may be due to an increased frequency of dynamical interactions (see, e.g., Zepf \& Whitmore 1993; Bettoni \& Fasano 1993; Mendes de Oliveira \& Hickson 1994; Pildis, Bregman, \& Schombert 1995). However, none of these observations indicate that a large fraction of the galaxies is strongly affected by their location in Hickson groups (Zepf 1995). The absence of evidence for a large fraction of ongoing mergers might be understood if the Hickson sample is composed of groups in a range of dynamical states (Ribeiro et al. 1997).

The slope of the faint end of the luminosity function for galaxies in our Hickson group sample we find here $(\alpha \simeq$ 
$-1.0)$ is consistent with our earlier estimate based on galaxy counts in the region of the groups and a statistical background correction (Ribeiro et al. 1994). However, as reviewed by Hickson (1997), some other analyses have suggested a depletion of faint galaxies in Hickson groups. This work is different than other surveys in two significant ways. First, our surveys go much deeper and therefore provide better leverage on the slope at the faint end. Second, by studying galaxies much fainter than those on which Hickson based his compact group selection, we avoid many of the potential biases associated with this selection.

A result related to this latter point is that we find that the faint galaxies are more diffusely distributed than the bright galaxies originally selected by Hickson. Thus, a study of the luminosity function restricted to the area on the sky that encloses the bright galaxies in the group will systematically underestimate the number of faint galaxies. The effect on the luminosity function can be significant, as we find that the average pairwise radius of the faint galaxies is about twice that of the bright galaxies.

The wider spatial distribution of the faint galaxies versus the bright galaxies can result from two different effects. One possibility is the bias inherent in selecting for a compact arrangement of bright galaxies in the plane of the sky. Clearly, this favors situations in which the bright galaxies are aligned to enhance their surface density. However, since the fainter galaxies are not part of the selection process, they are not biased in this way. They therefore may provide a truer representation of the extent of the group. It is also possible that the more diffuse distribution of faint galaxies arises from mass segregation. Although there is little evidence for such an effect in any other system of galaxies, it is difficult to choose between these two explanations solely on the basis of the available data. We note that in either case the true spatial extent of the Hickson groups is underestimated by a factor of several if only the bright galaxies are studied.

We are grateful to Huan Lin for providing the results of the Las Campanas Redshift Survey luminosity function study in digital form. This Letter benefited from discussions with Ann Zabludoff and from the suggestions of an anonymous referee. S. E. Z. acknowledges support from NASA through grant HF1055.01-93A awarded by the Space Telescope Science Institute (STScI), which is operated by the Association of Universities for Research in Astronomy, Inc., for NASA under contract NAS5-26555. A. L. B. Ribeiro acknowledges the support of the CAPES. We are grateful for the support of the Cerro Tololo Inter-American Observatory and STScI, at which the spectroscopy and digitized plate scans were obtained.

\section{REFERENCES}

Barnes, J. E. 1989, Nature, 338, 123

Bernstein, G. M., Nichol, R. C., Tyson, J. A., Ulmer, M. P., \& Wittman, D. 1995, AJ, 110, 1507

Bettoni, D., \& Fasano, G. 1993, 105, 1291

Colless, M. 1988, MNRAS, 237, 799

de Carvalho, R. R., Ribeiro, A. L. B., Capelato, H. V., \& Zepf, S. E. 1997, ApJS, 110, 1

de Carvalho, R. R., Ribeiro, A. L. B., \& Zepf, S. E. 1994, ApJS, 93, 47

De Propis, R., Pritchet, C. J., Harris, W. E., \& McClure, R. D. 1995, ApJ, 450,534

Driver, S. P., Phillips, S., Davies, J. I., Morgan, I., \& Disney, M. J. 1994, MNRAS, 268, 393

Dressler, A. 1978, ApJ, 223, 765

Gaidos, E. J. 1997, AJ, 113, 117

Hickson, P. 1982, ApJ, 255, 382 1997, ARA\&A, in press

Hickson, P., Kindl, E., \& Aumann, J. 1989, ApJS, 70, 687

Hickson, P., Kindl, E., \& Huchra, J. 1988, ApJ, 331, 64

Hickson, P., Mendes de Oliveira, C. M., Huchra, J. P., \& Palumbo, G. G. C. 1992, ApJ, 399, 353

Jeffreys, W. H., Fitzpatrick, M. J., \& McArthur, B. E. 1988, Celest. Mech., 41,39

Lin, H., et al. 1996a, ApJ, 471, 617

1996b, ApJ, 464, 60
Lopez-Cruz, O., Yee, H. K. C, Brown, J. P., Jones, C., \& Forman, W. 1997, ApJ, 475, L97

Loveday, J., Maddox, S. J., Efstathiou, G., \& Peterson, B. A. 1995, 442, 457

Loveday, J., Peterson, B. A., Efstathiou, G., \& Maddox, S. J. 1992, ApJ, 390, 338

Lugger, P. M. 1986, ApJ, 303, 555

Marzke, R. O., Geller, M. J., Huchra, J. P., \& Corwin, H. G. 1994a, ApJ, 428, 43 1994b, AJ, 108, 437

Mendes de Oliveira, C. M., \& Hickson, P. 1991, ApJ, 380, 30 1994, ApJ, 427, 684

Oemler, A. 1974, ApJ, 194, 1

Pildis, R. A., Bregman, J. N., \& Schombert, J. M. 1995, AJ, 110, 149

Ribeiro, A. L. B., de Carvalho, R. R., Capelato, H. V., \& Zepf, S. E. 1997, ApJ, submitted

Ribeiro, A. L. B., de Carvalho, R. R., \& Zepf, S. E. 1994, MNRAS, 267, L13

Sandage, A., Binggeli, B., \& Tammann, G. A. 1985, AJ, 90, 1759

Schechter, P. L. 1976, ApJ, 203, 297

Sulentic, J. W., \& Rabaca, C. R. 1994, ApJ, 429, 531

White, S. D. M., Davis, M., Efstathiou, G., \& Frenk, C. S. 1987, Nature, 330, 351

Zepf, S. E. 1993, ApJ, 407, 448

1995 , in Groups of Galaxies, ed. O.-G. Richter \& K. Borne (San Francisco: ASP), 135

Zepf, S. E., \& Whitmore, B. C. 1993, ApJ, 418, 72 